\title{
Magnetism and Kondo Effect in Cerium and Uranium Compounds
}

\author{
B. Coqbuin \\ Laboratoire de Physique des Solides, UMR 8502-CNRS \\ Université Paris-Sud, 91405 Orsay, France
}

\begin{abstract}
A brief review of the Kondo lattice problem is presented, with a special emphasis on the underscreened Kondo effect in ferromagnetic-Kondo uranium compounds and on the spin glass-Kondo competition in disordered heavy fermion cerium alloys.
\end{abstract}

PACS numbers: 75.20.Hr, 75.30.Mb, 05.50.+q, 64.60.Cn

\section{Introduction}

The Kondo effect has been observed experimentally in cerium or ytterbium impurities systems and a heavy fermion behavior has been characterized at low temperatures by very large values of the electronic specific heat constant and of the $T^{2}$ law of the electrical resistivity. An exact solution of the single impurity Kondo effect was theoretically determined roughly 30 years ago and the localized magnetic moment is completely screened by the conduction electron spins at $T=0$. In the case of a lattice, there is a strong competition between the Kondo effect on each site and the magnetic order arising from the Ruderman-Kittel-Kasuya-Yosida (RKKY) interaction between rare-earth atoms on different lattice sites, which has been firstly described by the well-known "Doniach diagram".

We will present here a very brief review of recent works on the normal and underscreened Kondo lattice and also on the spin glass-Kondo interaction. Detailed reviews can be found in Refs. [1-4].

\section{The Kondo lattice}

In order to describe the Kondo lattice, we use the Hamiltonian [5, 6]:

$$
H=\sum_{\boldsymbol{k} \sigma}\left(\epsilon_{\boldsymbol{k}}-\mu\right) c_{\boldsymbol{k} \sigma}^{\dagger} c_{\boldsymbol{k} \sigma}+\sum_{i \sigma \alpha} E_{0} n_{i \sigma}^{f_{\alpha}}+J_{\mathrm{K}} \sum_{i} \boldsymbol{S}_{i} \boldsymbol{\sigma}_{i}+\frac{1}{2} J_{H} \sum_{i j} \boldsymbol{S}_{i} \boldsymbol{S}_{j}
$$

The third term is the intrasite Kondo interaction described by the coupling constant $J_{\mathrm{K}}$ and the fourth term is the intersite magnetic interaction described by $J_{H}$. The localized spins are either $\boldsymbol{S}=1 / 2$ for the normal Kondo lattice [3] or 
$\boldsymbol{S}=1$ for the underscreened Kondo lattice (with $\alpha=1,2$ to describe the configuration $f^{2}$ ) [4]. There exists a strong competition between the Kondo effect and the magnetic order at low temperatures in cerium and ytterbium compounds: Cerium compounds are separated into those which do not order at low temperatures and have a very large electronic specific heat constant $\gamma\left(\right.$ like $\left.\mathrm{CeAl}_{3}\right)$ and those which order magnetically and present a relatively smaller heavy fermion behavior at low temperatures (like $\mathrm{CeAl}_{2}$ ); these compounds order generally in an antiferromagnetic order with a Néel temperature generally smaller than $10 \mathrm{~K}$. Our mean field treatment can account for the Kondo effect which increases with increasing $J_{\mathrm{K}}$ and decreases with increasing $J_{H}$ or a decreasing number of conduction electrons [3].

On the other hand, the situation of uranium compounds is quite different and complex. The $5 f$ electrons are much less localized than the $4 f$ electrons of rare-earths, as seen for example in the actinide series where magnetism occurs only for curium in the middle of the series. However, uranium compounds can present a great variety of different behaviors and in particular, some of them, like $\mathrm{UTe}, \mathrm{UCu}_{0.9} \mathrm{Sb}_{2}$ or $\mathrm{UCo}_{0.5} \mathrm{Sb}_{2}$ present a ferromagnetic ordering at very large Curie temperatures (equal respectively to $T_{\mathrm{c}}=102 \mathrm{~K}, 113 \mathrm{~K}$, and $64.5 \mathrm{~K}$ ) and show a $\log T$ Kondo-type decrease in the resistivity above $T_{\mathrm{c}}[7]$.

We have recently developed the underscreened Kondo lattice (UKL) model starting from the Hamiltonian (1) with two $5 f$ electrons [4] and we have computed in the mean field approximation the magnetizations of the localized and conduction electrons and the Kondo correlations for the two spin directions. For a large $J_{\mathrm{K}}$ value, we have obtained a Kondo-ferromagnetic state at very low temperatures below the Curie temperature $T_{\mathrm{c}}$, then a Kondo state between $T_{\mathrm{c}}$ and the Kondo temperature $T_{\mathrm{K}}$ and finally a paramagnetic state above $T_{\mathrm{K}}$. Then, we have derived the phase diagram giving $T_{\mathrm{c}}$ and $T_{\mathrm{K}}$ versus $J_{\mathrm{K}}$ and we have shown that $T_{\mathrm{K}}$ is increasing abruptly above a critical value $J_{\mathrm{K}}^{\mathrm{c}}$, while $T_{\mathrm{c}}$ is always non-zero but increases slowly above $J_{\mathrm{K}}^{\mathrm{c}}$ and remains there smaller than $T_{\mathrm{K}}$. This "ferromagnetic-Kondo" diagram is completely different from the Doniach diagram derived for cerium compounds. Thus, the UKL model explains the behavior of ferromagnetic Kondo uranium compounds, including also $\mathrm{UNiSi}_{2}$ with $T_{\mathrm{c}}=95 \mathrm{~K}[8]$ or even a neptunium compound $\mathrm{NpNiSi}_{2}$ [9], which becomes ferromagnetic at $T_{\mathrm{c}}=51.5 \mathrm{~K}$ and presents a Kondo behavior.

However, possible extensions of the UKL model would be very interesting to be developed, in order to describe the coexistence between the ferromagnetic order and a non-Fermi-liquid behavior, as observed in $\mathrm{URu}_{2-x} \mathrm{Re}_{x} \mathrm{Si}_{2}$ compounds [10] or the existence of a decrease in $T_{\mathrm{c}}$ versus $J_{\mathrm{K}}$ down to eventually a quantum critical point. Finally, the question of the localization of the $5 f$ electrons is worth to be still studied. The $5 f$ electrons are well localized in UTe and itinerant in US, while they are intermediate in USe [11]. $T_{\mathrm{c}}$ is initially increasing with pressure in UTe, in good agreement with the present UKL model, while it decreases at high pressure in UTe and decreases always with pressure in US; this pressure 
decrease of $T_{\mathrm{c}}$ is certainly connected with the delocalization of the $5 f$ electrons and a complete treatment of the underscreened Anderson lattice model would be necessary to describe firstly the Kondo regime as in the UKL model and then a continuous transition to an intermediate valence regime.

\section{The spin glass-Kondo interaction}

We have also studied in detail the Hamiltonian (1) with the same Kondo term, but with a modified fourth term in order to describe the spin-glass character; this last term is given now by a quantum Ising interaction between the $z$-components of the localized spins and the exchange integrals are taken as ramdomly distributed with a Gaussian distribution like in the Sherrington-Kirkpatrick model. The purpose of this work was to account for the experimental phase diagrams of cerium systems such as $\mathrm{CeCu}_{1-x} \mathrm{Ni}_{x}$ or $\mathrm{Ce}_{2} \mathrm{Au}_{1-x} \mathrm{Co}_{x} \mathrm{Si}_{3}$ disordered alloys where the Kondo, spin-glass, and magnetically ordered phases are observed. Different approaches are described in Ref. [1] and we have initially obtained a phase diagram showing first a spin-glass phase and then a Kondo phase with increasing $J_{\mathrm{K}}$ [12]; important improvements were considered in order firstly to obtain a more complex phase diagram with a ferromagnetic or an antiferromagnetic phase occurring at low temperatures for smaller $J_{\mathrm{K}}$ values and also to derive a quantum critical point for the magnetic phase [13]. Many papers are available on this approach and the references can be found in Refs. [1, 13]. A very careful experimental study of $\mathrm{CeCu}_{0.6} \mathrm{Ni}_{0.4}$ yields a percolative transition with decreasing temperature from a cluster-glass state with ferromagnetic correlations to a ferromagnetic clustered state and recent theoretical simulations can reproduce satisfactorily the experimental situation [14]. Moreover, the Kondo-spin glass-ferromagnetism coexistence in disordered cerium compounds has been also recently studied by introducing a generalization of the Mattis model which represents an interpolation between ferromagnetism and a highly disordered spin glass [15].

\section{Conclusions}

We have presented here a summary of recent works which have been performed in several directions. After the study of the normal Kondo lattice, we have developed the underscreened Kondo lattice which can explain the behavior of some uranium ferromagnetic Kondo compounds and the spin glass Kondo-magnetic order interaction which can account for the phase diagrams of disordered cerium alloys. Detailed calculations and experimental data can be found in the references cited below.

\section{References}

[1] B. Coqblin, M.D. Nunez-Regueiro, A. Theumann, J.R. Iglesias, S.G. Magalhaes, Philos. Mag. 86, 2567 (2006).

[2] B. Coqblin, AIP Conf. Proc. 846, 3 (2006). 
[3] B. Coqblin, C. Lacroix, M.A. Gusmão, J.R. Iglesias, Phys. Rev. B 67, 064417 (2003).

[4] N.B. Perkins, M.D. Nunez-Regueiro, B. Coqblin, J.R. Iglesias, Phys. Rev B 76, 125101 (2007).

[5] P. Coleman, N. Andrei, J. Phys., Condens. Matter 1, 4057 (1989).

[6] J.R. Iglesias, C. Lacroix, B. Coqblin, Phys. Rev. B 56, 11820 (1997).

[7] J. Schoenes, J. Less-Common Met. 121, 87 (1986).

[8] D. Kaczorowski, Solid State Commun. 99, 949 (1998).

[9] E. Colineau, F. Wastin, J.P. Sanchez, J. Rebizant, presented at the 37èmes Journées des Actinides, Sesimbra (Portugal) 2007.

[10] E.D. Bauer, V.S. Zapf, P.C. Ho, N.P. Butch, E.J. Freeman, C. Sirvent, M.B. Maple, Phys. Rev. Lett. 94, 046401 (2005).

[11] Q.G. Sheng, B.R. Cooper, J. Magn. Magn. Mater. 164, 335 (1996).

[12] A. Theumann, B. Coqblin, S.G. Magalhaes, A.A. Schmidt, Phys. Rev. B 63, 054409 (2001).

[13] S.G. Magalhaes, F.M. Zimmer, B. Coqblin, J. Phys., Condens. Matter 18, 3479 (2006).

[14] N. Marcano, to appear in Phys. Rev. Lett. (2007).

[15] S.G. Magalhaes, J.C. Gomez Sal, J.I. Espeso, J.M. De Teresa, P.A. Algarabel, C. Paulsen, J.R. Iglesias, Phys. Rev. B 74, 014427 (2006). 